\title{
Remote hand: Hand-centered peripersonal space transfers to a disconnected hand avatar
}

Daisuke Mine $^{1 *}$, Kazuhiko Yokosawa ${ }^{1}$

${ }^{1}$ Department of Psychology, The University of Tokyo, Tokyo, Japan

* Corresponding author

Email: mine@1.u-tokyo.ac.jp

\begin{abstract}
The space surrounding our body is called peripersonal space (PPS). It has been reported that visuo-tactile facilitation occurs more strongly within PPS than outside PPS. Furthermore, previous research has revealed several methods by which PPS can be extended. The present study provides the first behavioral evidence of the transfer of PPS in a virtual environment by a novel technique. PPS representation was investigated using a remote-controlled hand avatar presented far from their body in a virtual environment. Participants showed strongest visuo-tactile facilitation at the far space around the remote hand and no facilitation at the near space around the real hand, suggesting that PPS transfers from near the body to the space around the hand avatar. The present results extend previous findings of the plasticity of PPS and demonstrate flexibility of PPS representation beyond the physical and anatomical limits of body representation.
\end{abstract}

Keywords: Peripersonal space, Spatial perception, Body representation, Virtual reality 


\section{Introduction}

The space immediately surrounding the body, where an agent can physically interact with the environment through the body, is called peripersonal space (PPS) (Rizzolatti, Fadiga, Fogassi, \& Gallese, 1997). Numerous studies in neurophysiology, neuropsychology, and psychophysics have provided evidence of specific brain activity that encodes the spatial representation surrounding the body. PPS is encoded by multi-modal neurons in the frontoparietal network, including premotor cortex and posterior parietal cortex. These multi-modal neurons respond to visual or auditory stimuli presented in the space surrounding the specific body part where tactile inputs are simultaneously delivered. Intensity of the response of these multi-modal neurons usually depends on the Euclidean distance from the tactile receptive field of the multi-modal neurons that are anchored to a specific body part; therefore these multi-modal neurons are believed to represent a body-part centered reference frame (Colby, 1998). Based on these neural activities, PPS is thought to play a functional role in guidance of visuo-motor actions by specific body parts (di Pellegrino \& Làdavas, 2015).

Individuals perform various visuo-motor actions not only with their own body parts but also with hand-held tools. Iriki and colleagues reported that in macaque monkeys, the visual receptive field of neurons that represent hand-centered PPS (HPPS) extends toward the tool tip when individuals actively use tools (Iriki, Tanaka, \& Iwamura, 1996). Since Iriki et al.'s report, much neuropsychological and behavioral research has found supporting evidence that tool-use extends HPPS in human (Holmes, Calvert, \& Spence, 2004; Farnè, Iriki, \& Làdavas, 2005; Farnè et al., 2007; Longo \& Lourenco, 2006; see Serino, 2019, for a review).

Previous studies have investigated HPPS representation when individuals control their own hand or hand-held tools. However, with recent technological developments, we can experience controlling and using disconnected artifacts. Particularly in a virtual environment, where there are no physical limitations such as those in the real environment, individuals can use avatars, often surreally modified, as their bodies. PPS representations when individuals control these remotely controlled avatars or modified bodies have not yet been fully investigated. A previous research revealed that HPPS appears around a hand avatar that is disconnected and presented far from the body in a virtual environment; however, HPPS representation was investigated only in the space around the disconnected hand avatar, but not around the participant's real hand or in the intermediate space between the participant's real hand and the disconnected hand (Mine \& Yokosawa, 2020). In the present study, we investigated HPPS representation when individuals experienced a disconnected hand avatar that could be controlled through the movement of their own hand in a virtual environment.

We focused on two main questions. The first is whether HPPS appears around the disconnected hand avatar. Mine and Yokosawa (2020) used a line-bisection task to reveal that HPPS can appear around the disconnected hand (see Jewell \& McCourt, 2000; McCourt \& Garlinghouse, 2000; Varnava, McCarthy, \& Beaumont, 2002). The linebisection task has been used as a measurement of HPPS, but because the neural mechanisms relevant to the task are not yet fully understood, it is unknown whether performance of the line-bisection task is really based on the activation of multi-modal neurons. The first purpose of the present study was therefore to replicate the result of Mine and Yokosawa (2020) using a multi-modal facilitation task that is assumed to reflect the activation of multimodal neurons. If HPPS does appear around the disconnected hand avatar, the second question is whether HPPS 
emerges around the hand avatar itself or instead arises from the space around the position of the participant's real hand and then extends to the hand avatar. Previous studies of HPPS representation during tool use have arrived at two different conclusions about HPPS modulation. Farnè, Serino, and Làdavas (2007) have reported that HPPS extends along a tool held by a participant. On the other hand, Holmes et al. (2004) suggested that multi-modal facilitation can be observed near the hand or at the tool tip, but not at the middle of the tool shaft. We investigate the intermediate space between the disconnected avatar and the real hand, when nothing is presented in the space.

Given the evidence summarized above, four alternative hypotheses can be proposed for HPPS representation when participants use the disconnected hand. The first hypothesis, the extension model, is that HPPS extends from the space around the participant's real hand to the space around the disconnected hand (Fig 1a). The second hypothesis, the addition model, is that HPPS appears around the disconnected hand in a distinct location, in addition to the space around the participant's real hand (Fig 1b). The third hypothesis, the transfer model, is that HPPS emerges around the disconnected hand while HPPS around the participant's real hand disappears (Fig 1c). The fourth hypothesis, the no-change model, is that HPPS representation does not change when the disconnected hand is used (Fig 1d). If the results of Mine and Yokosawa (2020) can be replicated, HPPS should appear around the disconnected hand; however, Mine and Yokosawa (2020) did not investigate HPPS representation around the real hand or around the intermediate space between the real hand and disconnected hand.

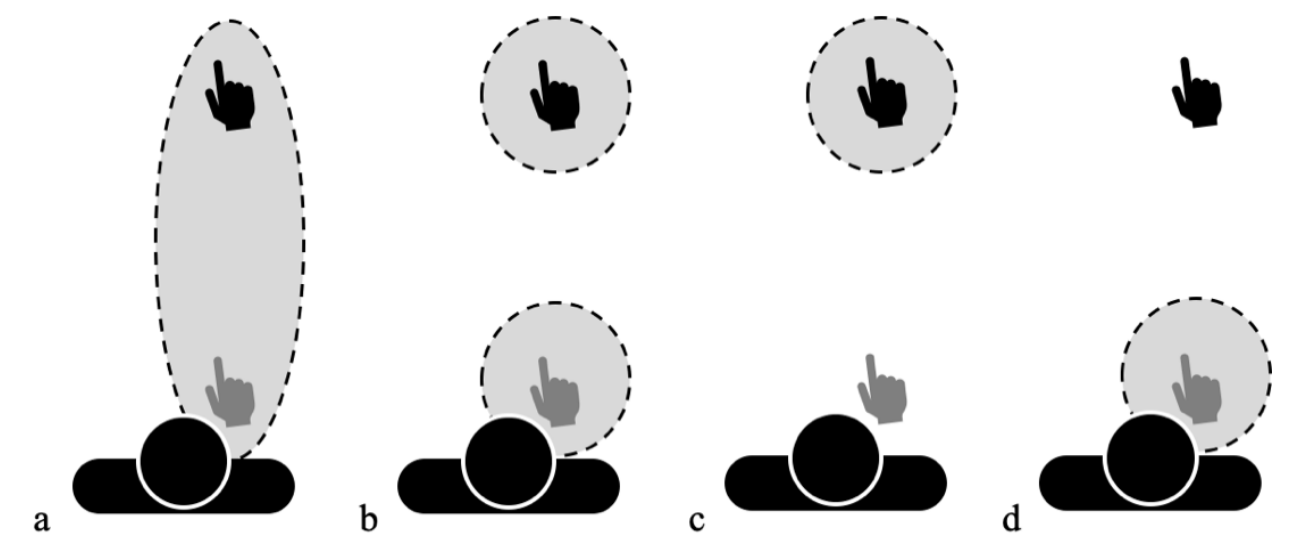

Fig 1: Hypotheses of HPPS representation during disconnected hand use. Black hands indicate disconnected hand avatars and grey hands indicate participants' real hands. (a) Extension model, with HPPS extending to the disconnected hand from the position of the participant's real hand. (b) Addition model, with HPPS emerging around the disconnected hand in addition to the participant's real hand. (c) Transfer model, with HPPS emerging around the disconnected hand and disappearing around the participants' real hand. (d) No-change model, with HPPS representation unchanged when the disconnected hand is used.

\section{Method}

\subsection{Participants}

We recruited a total of 53 healthy participants as paid volunteers, with 28 (14 female) participating in Experiment 1 and 25 (13 female) in Experiment 2. Because no other studies investigated HPPS representation 
around a disconnected hand, we calculated the sample size of Experiment 1 on the basis of a desired power of 0.8 and the assumed effect size $(f=.2)$ of an interaction between visual stimulus position and hand position. A sample size of 25 for Experiment 2 was calculated from the effect size of multi-modal facilitation in Experiment 1 . All participants were right-handed and had normal or corrected-to-normal stereo vision. All participants gave written informed consent before participating in the experiments. The experiments were approved by the ethics committee of the Department of Psychology of the University of Tokyo. All procedures described below were approved by the same ethics committee and conducted according to the principles and guidelines of the Declaration of Helsinki.

\subsection{Materials and stimuli}

A participant saw a virtual environment through a head mounted display (HMD: HTC VIVE, displaying a stereoscopic image with a resolution of $2160 \times 1200$ ). A virtual world was developed using Unity3D and run on a Windows PC (Level Infinity by iiyama: Intel core i7-7700HQ at $2.8 \mathrm{GHz}, 16 \mathrm{~GB}$ RAM, and NVIDIA GeForce GTX 1060). In the virtual environment, the participant saw a virtual hand avatar from a first person perspective, and the position and movement of the participant's hand were tracked using the HTC VIVE controller.

In both experiments, we used a multi-modal facilitation task as a measurement of HPPS representation (see Serino, Bassolino, Farnè, \& Làdavas, 2007; Noel et al., 2020). In the multi-modal facilitation task, participants are instructed to react to tactile stimuli delivered to the hand while ignoring visual or auditory stimuli concurrently presented at any distance from the hand, and participants typically react faster to the tactile stimulus when the visual or auditory stimulus is presented near the body than when it is presented far from the body (see Macaluso \& Maravita, 2010, for a review). Serino, Canzoneri, and Avenanti (2011) found that inhibitory transcranial magnetic stimulation over intra-parietal sulcus (IPS) and ventral premotor cortex (vPMC) reduced facilitation in reaction time for tactile stimuli in the multi-modal facilitation task. Because IPS and vPMC form the main portions of the frontoparietal network where multi-modal neurons are observed, the results of Serino et al. (2011) provide strong evidence that the facilitation in reaction time observed in the multi-modal facilitation task reflects activation of multi-modal neurons. In the present study, tactile stimuli (vibrations of duration $200 \mathrm{~ms}$ and frequency $80 \mathrm{~Hz}$ ) were delivered from the HTC VIVE controller held in the right hand. A white ball (diameter $10 \mathrm{~cm}$ ) was used as the visual stimulus in the virtual environment (Fig 2). Although looming visual (or auditory) stimuli have been used in most previous investigations of HPPS, multi-modal facilitation effects and temporal expectancy effects may be confounded with looming stimuli (Kandula, Van der Stoep, Hofman, \& Dijkerman, 2017). To eliminate temporal expectancy effects, we used static visual stimuli in the present study (see Experiment 2 of Noel et al., 2020). A white ball was presented at $120 \mathrm{~cm}$ (D1), $80 \mathrm{~cm}$ (D2), or $30 \mathrm{~cm}$ (D3) from the participant's body, and the onset of the appearance of the white ball was identical to the onset of tactile stimulation on the participant's right hand. Participants were instructed to press a key with their left index finger as fast as possible when they perceived tactile stimulation, while ignoring the visual stimuli simultaneously presented. In a baseline condition, participants responded to the tactile stimulus while no visual stimulus was presented. The experimental design referred to the methods of previous studies (Serino et al., 2017; Noel et al., 2020). Mean reaction time to the baseline condition was calculated for each participant to eliminate variation between participants according to ability to respond to 
tactile stimuli.

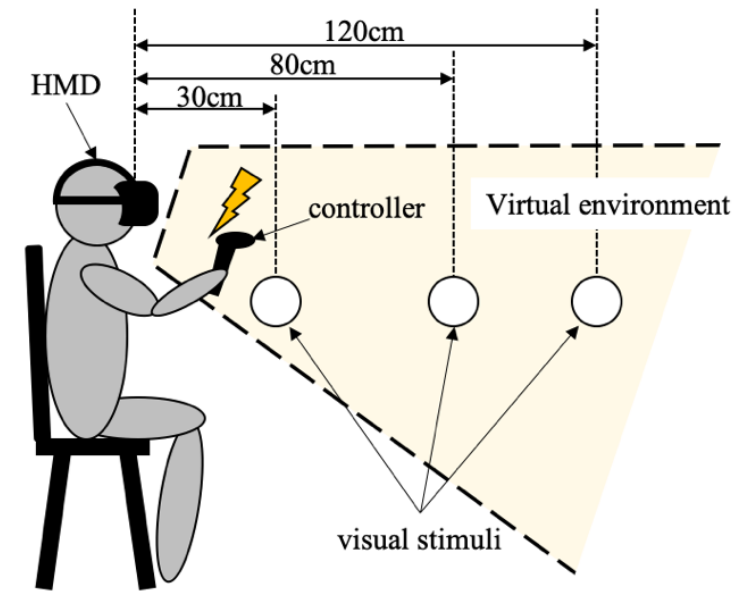

a

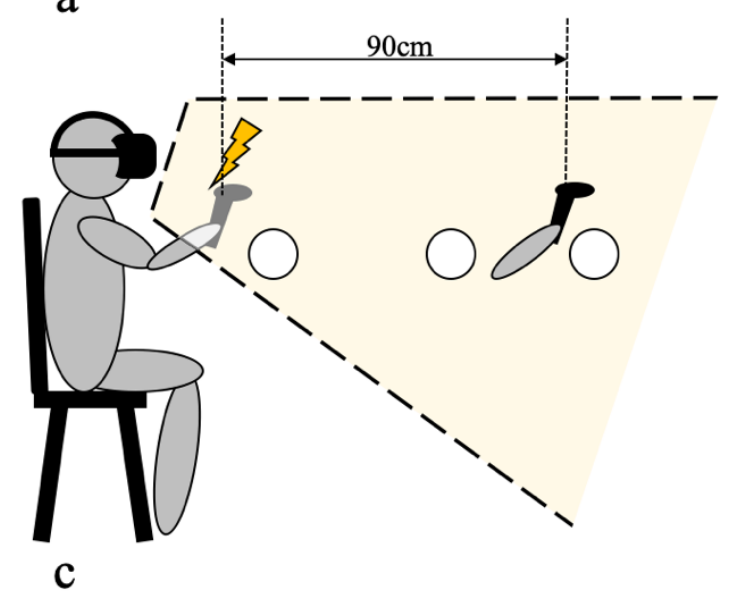

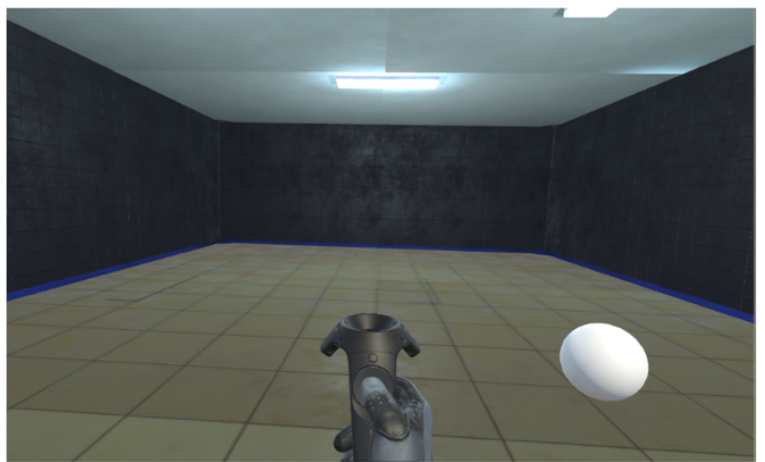

b

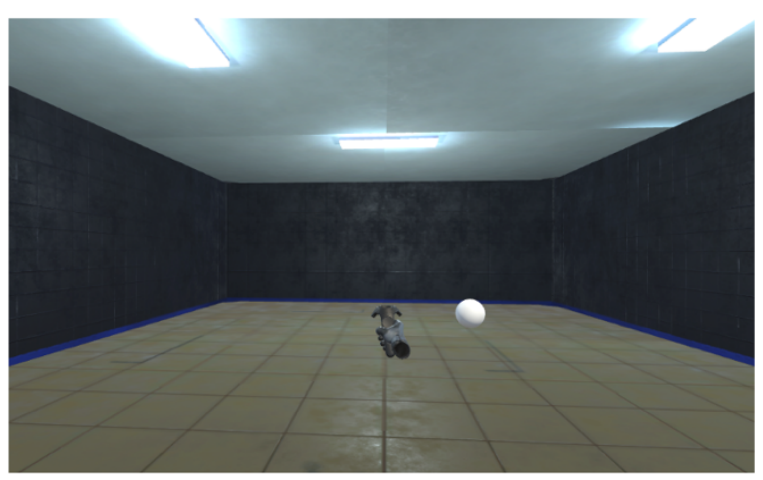

d

Fig 2: Experimental setups of Experiment 1. The top two figures $(a, b)$ represent the condition in which the hand avatar was presented at the normal position of the participant's real hand. The bottom two figures (c, d) represent the condition in which the hand avatar was presented at the far position from the participant's body. The left two figures (a, c) represent overall views of the experimental setups, and the dotted lines designate the virtual environment that the participant saw through the HMD. The right two figures (b, d) show the virtual environment from the participant's view. The participant saw the right hand and controller in the virtual environment. When the virtual hand was presented far from the body, no hand was presented at the position of the participant's real hand.

\section{Experiment 1}

\subsection{Procedure}

A participant sat at one end of a table in the lab space and was instructed to adjust the fit of the HMD and to handle the controller with the right hand. The participant's head was then positioned on a chin rest attached to the table. The chin rest was used to prevent participant head movements during the experiment. There are two conditions of hand position. In one condition, the hand avatar was presented at the position of the participant's real 
hand, and in the other, the hand avatar was presented $90 \mathrm{~cm}$ in front of the position of the participant's real hand. When entering the virtual environment, the participant saw a virtual room which was empty except for the hand avatar and a white cylinder. The participant was asked to reach for the cylinder with the virtual right hand. The reaching task was included to make participants recognize that they could actively control the hand avatars. After the reaching task, the scene changed to the following reaction task. A fixation cross appeared in front of the participant, and the participant was asked to fixate on the cross position during the reaction task. After the fixation cross disappeared, tactile and visual stimuli were presented at a latency of either $1250 \mathrm{~ms}, 1500 \mathrm{~ms}, 1750 \mathrm{~ms}$, or $2000 \mathrm{~ms}$ (randomized). The participant was instructed to react to the tactile stimulus delivered to the right hand by pressing the Enter key using the left index finger, while ignoring the visual stimulus. After the participant reacted to the tactile stimulus, the scene changed to the reaching task of the next trial. Each participant performed a total of 192 trials: 2 hand positions (near, far) $\times 4$ stimulus positions $(30 \mathrm{~cm}, 80 \mathrm{~cm}, 120 \mathrm{~cm}$, no stimulus $) \times 24$ repetitions.

\subsection{Statistical analyses}

We defined the multi-modal facilitation effect as the difference between reaction time to the unimodal stimuli and reaction time to the multi-modal stimuli. A positive value indicates facilitation of the reaction to the tactile stimulus caused by the presentation of visual stimuli. The multi-modal facilitation effect was calculated for each participant for each visual stimulus position. Outliers were defined as reaction times that exceeded three times the standard deviation from each participant's mean reaction time for each hand position condition and were excluded from analysis. A two-way repeated measures ANOVA (visual stimulus position $\times$ hand position) and subsequent analyses were conducted using the statistical software package R.

\subsection{Results}

Of the 28 participants, one was unable to complete the experimental procedure because of equipment errors. Therefore, we excluded these data and analyzed the data of the remaining 27 participants. The results are shown in Fig 3. A two-way repeated measures ANOVA revealed a significant interaction between visual stimulus position and hand position $\left(F(2,52)=10.53, p<.001, \eta_{p}^{2}=.288\right)$, and simple effect analyses of visual stimulus position for each hand position revealed significant simple effects of visual stimulus position in both the near-hand condition and the far-hand condition (near: $F(2,52)=9.33, p<.001, \eta_{p}^{2}=.264$; far: $F(2,52)=3.72, p=.031, \eta_{p}^{2}$ $=.125)$. Post-hoc analyses using Holm's method for each pair of conditions revealed a significant difference at D3 compared to D1 and D2 for the near-hand condition (D3 versus D1: $t(26)=3.95, p<.001, d=.760$; D3 versus D2: $t(26)=2.46, p=.021, d=.473)$. The difference between D1 and D2 was also significant $(t(26)=2.13, p=.043, d$ $=.411$ ). These results indicate that the multi-modal facilitation effect was significantly greater in the space near the participant's hand than in the farther space, as many prior studies have shown. More interestingly, for the far-hand condition, the multi-modal facilitation effect was significantly higher at D1 compared to D2 and D3, and the difference between D2 and D3 was not significant (D1 versus D2: $t(26)=2.96, p=.0065, d=.569$; D1 versus D3: $t(26)=2.45, p=.021, d=.471 ; \mathrm{D} 2$ versus D3: $t(26)=0.49, p=.63, d=.094)$. This pattern of results represents a 
successful replication of Mine and Yokosawa (2020) and further suggests that, when the hand avatar was placed in the space far from the participant's body, HPPS emerged around the hand avatar. Furthermore, simple effects analyses of hand position for each visual stimulus position revealed significant effects at D1 and D3 but not at D2 $\left(\mathrm{D} 1: F(1,26)=4.72, p=.039, \eta_{p}^{2}=.154\right.$; D2: $F(1,26)=1.44, p=.24, \eta_{p}^{2}=.052$; D3: $F(1,26)=7.67, p=.010$, $\left.\eta_{p}^{2}=.228\right)$. These results suggest that when the hand avatar was placed in the space far from the participant's body, HPPS emerged in the far space and disappeared from the near space, as if HPPS had been transferred from the space near the body to the space around the hand avatar.

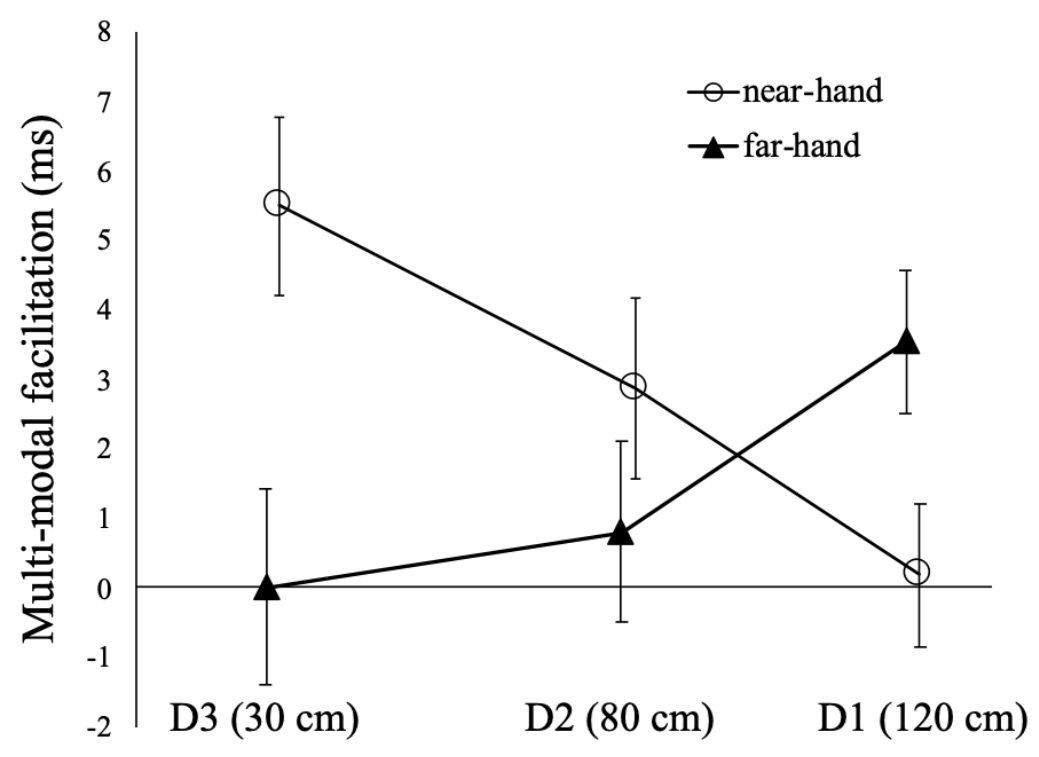

Visual stimulus position

Fig 3: Multi-modal facilitation effects for far-hand (black triangles) and near-hand (circles) positions at three visual stimulus positions. Error bars show the standard error.

\subsection{Discussion}

The main goal of Experiment 1 was to investigate HPPS representation when individuals used hand avatars that were presented far from the body. When the hand avatar was presented in far space, multi-modal facilitation was higher at the far space than at the normal (near-hand) position. Thus, as previously shown by Mine and Yokosawa (2020), HPPS appeared around the disconnected hand avatar. Furthermore, multi-modal facilitation was higher at the far space than at the middle space, suggesting that HPPS emerged around the disconnected hand avatar rather than extended from the space around the participant's real hand. More interestingly, HPPS around the participant's real hand position disappeared in the far-hand condition. Although previous studies have shown examples of PPS extension from a real body part (or from the full body) when some tools are used, disappearance of HPPS around the real hand has not been previously reported. A possible interpretation of this phenomenon is that HPPS transferred from the space around the real hand position to the space around the far hand avatar. However, there is a critical difference between the previous studies and the present one. In the far hand condition in the present study, the hand avatar at the position of the real hand was not displayed, while participants were able to see 
their real hand holding tools in the previous studies. Therefore, it is possible that HPPS disappeared simply because no hand was presented at the near position. To evaluate this possibility, in Experiment 2 we investigated HPPS representation when no hand was presented in the virtual environment.

\section{Experiment 2}

\subsection{Procedure}

Experiment 2 included two conditions of hand visibility instead of hand position, as in Experiment 1. The visible hand condition was identical to the near-hand condition in Experiment 1, in which the hand avatar was presented at the position of the participant's real hand. In the invisible hand condition, no hand was presented in the virtual environment. The procedure of Experiment 2 was almost identical to that in Experiment 1, with the following exceptions. The reaching task that was performed before each reaction time trial in Experiment 1 was not performed in Experiment 2 because it was impossible to reach for the target using an invisible hand. The purpose of the reaching task was to make participants recognize that they could actively control the disconnected hand, a procedure that did not make sense in Experiment 2. Further, the white ball was presented only at $30 \mathrm{~cm}$ from the body in order to focus on whether visibility of the hand affects HPPS representation in the space around the position of the participant's real hand. Thus, each participant performed a total of 96 trials: 2 hand visibilities (visible, invisible) $\times 2$ visual stimulus positions $(30 \mathrm{~cm}$, no stimulus $) \times 24$ repetitions.

\subsection{Statistical analyses}

The multi-modal facilitation effect was calculated for each participant using the procedure described in Experiment 1 (reaction time for unimodal stimuli minus reaction time for multi-modal stimuli). Outliers were defined as reaction times that exceeded three times the standard deviation from each participant's mean reaction time for each hand position and were excluded from analyses. We compared the multi-modal facilitation effect to zero in both visible and invisible hand conditions and then compared the strength of the multi-modal facilitation effect between these two conditions. To control the proportion of false positives, we adjusted $p$ values using Holm's method. All data were analyzed using the statistical software package R.

\subsection{Results}

We conducted three t-tests on the means shown in Fig 4. The first one-sample t-test revealed that multi-modal facilitation in the visible hand condition was significantly greater than zero $(t(24)=2.46, p=.021, d=.492)$. The second one-sample t-test revealed that multi-modal facilitation in the invisible hand condition was also significantly greater than zero $(t(24)=2.93, p=.0073, d=.586)$. The comparison of multi-modal facilitation between the visible and invisible hand conditions revealed no significant difference $(t(24)=0.72, p=.479, d=.144)$. Thus, a visual stimulus presented near the participant's hand facilitated the response to the tactile stimuli whether or not the hand 
was visually presented.

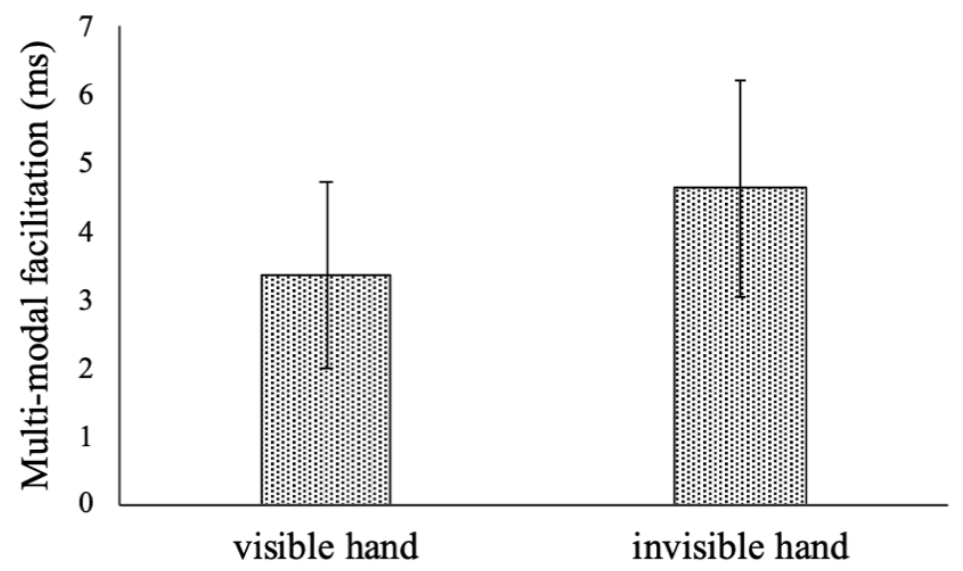

Fig 4: Multi-modal facilitation effect for visible and invisible hand conditions. Error bars show the standard error.

\subsection{Discussion}

Experiment 2 demonstrated that multi-modal facilitation could be observed in the space around the real hand position even when no hand was presented in the virtual environment, and this facilitation in the invisible hand condition was on the same level as the facilitation observed in the visible hand condition. This result suggests that visibility of the hand alone does not affect the HPPS representation.

This result is consistent with a previous study that suggested that visibility of the hand does not modulate the HPPS boundary (D’Angelo, Pellegrino, \& Frassinetti, 2017). Graziano (1999) found that response of macaque monkeys' multi-modal neurons was affected by arm position, even when the monkeys' arms were hidden from their view. Thus, proprioceptive information plays a significant role in detecting the position of specific body parts and appears to affect anchoring of HPPS representations to body parts even when there are no visual cues.

\section{General Discussion}

In Experiment 1, when a hand avatar in a virtual environment was placed far from the participant's body, HPPS emerged around the disconnected hand avatar and concurrently disappeared from the space around the participant's real hand position. Experiment 2 established that the disappearance of HPPS at the near space was not attributable to invisibility of the hand avatar at the participant's real hand position. These results suggest that HPPS transferred from the space where the real hand was located to the space around the disconnected hand. Although ample research has investigated the plasticity of PPS, especially HPPS, this transfer of PPS has not previously been reported.

Some research has suggested that spatial limits of body ownership reflect the PPS boundary (D. M. Lloyd, 2007; Blanke, Slater, \& Serino, 2015). Body ownership is known to be restricted by physical continuity or anatomical congruency (Perez-Marcos, Sanchez-Vives, \& Slater, 2012; Ehrsson, Spence, \& Passingham, 2004); however, the present results indicate that PPS can occur around a disconnected avatar. Therefore, spatial 
representation of PPS and spatial limitations of body ownership are not identically mapped, although the spatial limitations of ownership may be included in PPS.

A possible explanation for the transfer of HPPS from near to far is that HPPS reflects the reference frame required for visuo-motor action. When an individual performs a reaching action with their hand, the position of the target is described with reference to the position of the hand at first, and then the planned trajectory in the spatial reference frame is transformed into the movement of joints and muscles. In the present study, the movement of the disconnected hand avatar was perfectly synchronized with the movement of the participant's real hand, which was invisible to the participants; that is, if a participant wanted to move the disconnected hand avatar a specific distance in a specific direction, they moved their real hand the same distance in the same direction. If a reaching action was conducted with the disconnected hand avatar, the desired trajectory could be determined only from the relative position of the target and the hand avatar, without respect to the position of the real hand. Thus, a reference frame centered on the real hand was not needed to handle the disconnected hand avatar; that is why HPPS was transferred to the far space in the present study. As previous studies has revealed, multi-modal neurons located in frontoparietal network represents the hand (or upper arm) centered reference frame (Colby, 1998, for a review). Therefore, it is assumed that PPS centered on specific body part represents the space where they can interact with the environment through the movement of the body part.

Another possible explanation is that the disconnected hand avatar that was visibly presented overrode the invisible real hand. In Experiment 2, HPPS did not disappear around the real hand position even when no hand was presented; however, much previous research has revealed top-down effects of visual information on the perception of body part positions and HPPS representations. In a cross-modal congruency task, Pavani, Spence, and Driver (2000) demonstrated that visual cues presented near a fake rubber hand facilitated reaction to a tactile stimulus that was delivered on the participant's real but hidden hand. Lloyd, Morrison, and Roberts (2006) found that a potentially noxious visual stimulus near a fake rubber hand activated HPPS neurons. These studies suggest that topdown visual cues from a fake hand can override proprioceptive cues from the position of a real hand. However, these top-down effects were observed only when the position and posture of the fake hands met the anatomical limitations of the human body. In the present study, the disconnected hand avatar was presented $90 \mathrm{~cm}$ forward from the position of the participants' real hand, then the extent to which visual top-down effects influenced HPPS representation in the present paradigm remains unknown.

The present results extend previous findings about the flexibility of PPS by demonstrating the first behavioral evidence of the transfer of HPPS from the space near the body to a space far from the body. The present behavioral findings suggest two possible neural mechanisms that cannot be distinguished at this time and require further investigation: the visual receptive field of multi-modal neurons may transfer from near to far, or reinforcement may occur between synapses of multi-modal neurons and unimodal neurons that have receptive fields around the far space. It should be further noted that behavioral multi-modal facilitation of reaction time may be affected not only by the activation of multi-modal neurons induced by visuo-tactile inputs but also by confounds such as attention and response preparation. To confirm that HPPS can transfer from near to far, as suggested by the present results, future studies are needed to investigate the neural mechanisms relevant to HPPS modulation. 


\section{Conclusion}

In the present study, we investigated PPS representation when people handled a remote-controlled hand avatar that was disconnected and presented far from the body in a virtual environment. The results indicated that PPS transferred from near space, where the participant's hand was located, to far space around the hand avatar. This is the first demonstration of the transfer of PPS, and this result may suggest that PPS represents the reference frame required for visuo-motor action using specific body part including the disconnected hand avatar. A plenty of previous studies have revealed that PPS is flexibly spatially mapped in accordance with the agent's ability to move the body or some hand-held tools. The present results further the previous findings and offer suggestions about the fundamental nature of PPS, although future research must investigate whether behavioral results in a virtual environment reflect universal characteristics of PPS.

\section{Supporting information}

S1 Data. All relevant data to generate figures in our experiment.

\section{Acknowledgements}

This work was supported by Japan Society for the Promotion of Science [Grant numbers: 19H01490]. The funders had no role in study design, data collection, and analysis, decision to publish, or preparation of the manuscript.

\section{Author contributions}

Conceived and designed the experiment: DM KY. Performed the experiment: DM. Analyzed the data: DM. Wrote the paper: DM KY.

\section{Open Practices Statements}

All relevant data is available. None of the materials for the experiments reported here is available, and none of the experiments was preregistered.

\section{References}

Blanke, O., Slater, M., \& Serino, A. (2015). Behavioral, Neural, and Computational Principles of Bodily Self-

Consciousness. Neuron, 88(1), 145-166. https://doi.org/10.1016/j.neuron.2015.09.029

Colby, C. L. (1998). Action-oriented spatial reference frames in cortex. Neuron, 20(1), 15-24.

https://doi.org/10.1016/S0896-6273(00)80429-8

D’Angelo, M., Pellegrino, G. DI, \& Frassinetti, F. (2017). Invisible body illusion modulates interpersonal space. 
Scientific Reports, 7(1), 1-9. https://doi.org/10.1038/s41598-017-01441-9

di Pellegrino, G., \& Làdavas, E. (2015). Peripersonal space in the brain. Neuropsychologia, 66, 126-133. https://doi.org/10.1016/j.neuropsychologia.2014.11.011

Ehrsson, H. H., Spence, C., \& Passingham, R. E. (2004). That's my hand! Activity in premotor cortex reflects feeling of ownership of a limb. Science, 305(5685), 875-877. https://doi.org/10.1126/science.1097011

Farnè, A., Iriki, A., \& Làdavas, E. (2005). Shaping multisensory action-space with tools: Evidence from patients with cross-modal extinction. Neuropsychologia, 43(2 SPEC. ISS.), 238-248.

https://doi.org/10.1016/j.neuropsychologia.2004.11.010

Farnè, A., Serino, A., \& Làdavas, E. (2007). Dynamic size-change of peri-hand space following tool-use: Determinants and spatial characteristics revealed through cross-modal extinction. Cortex, 43(3), 436-443. https://doi.org/10.1016/S0010-9452(08)70468-4

Graziano, M. S. A. (1999). Where is my arm? The relative role of vision and proprioception in the neuronal representation of limb position. Proceedings of the National Academy of Sciences of the United States of America, 96(18), 10418-10421. https://doi.org/10.1073/pnas.96.18.10418

Holmes, N. P., Calvert, G. A., \& Spence, C. (2004). Extending or projecting peripersonal space with tools? Multisensory interactions highlight only the distal and proximal ends of tools. Neuroscience Letters, 372(1-2), 62-67. https://doi.org/10.1016/j.neulet.2004.09.024

Iriki, A., Tanaka, M., \& Iwamura, Y. (1996). Coding of modified body schema during tool use by macaque postcentral neurones. NeuroReport, 7(14), 2325-2330. https://doi.org/10.1097/00001756-199610020-00010

Jewell, G., \& McCourt, M. E. (2000). Pseudoneglect: A review and meta-analysis of performance factors in line bisection tasks. Neuropsychologia, 38(1), 93-110. https://doi.org/10.1016/S0028-3932(99)00045-7

Kandula, M., Van der Stoep, N., Hofman, D., \& Dijkerman, H. C. (2017). On the contribution of overt tactile expectations to visuo-tactile interactions within the peripersonal space. Experimental Brain Research, 235(8), 2511-2522. https://doi.org/10.1007/s00221-017-4965-9

Lloyd, D. M. (2007). Spatial limits on referred touch to an alien limb may reflect boundaries of visuo-tactile peripersonal space surrounding the hand. Brain and Cognition, 64(1), 104-109. https://doi.org/10.1016/j.bandc.2006.09.013

Lloyd, D., Morrison, I., \& Roberts, N. (2006). Role for human posterior parietal cortex in visual processing of aversive objects in peripersonal space. Journal of Neurophysiology, 95(1), 205-214. https://doi.org/10.1152/jn.00614.2005

Longo, M. R., \& Lourenco, S. F. (2006). On the nature of near space: Effects of tool use and the transition to far space. Neuropsychologia, 44(6), 977-981. https://doi.org/10.1016/j.neuropsychologia.2005.09.003

Macaluso, E., \& Maravita, A. (2010). The representation of space near the body through touch and vision. Neuropsychologia, 48(3), 782-795. https://doi.org/10.1016/j.neuropsychologia.2009.10.010

McCourt, M. E., \& Garlinghouse, M. (2000). Asymmetries of visuospatial attention are modulated by viewing distance and visual field elevation: Pseudoneglect in peripersonal and extrapersonal space. Cortex, 36(5), 715-731. https://doi.org/10.1016/S0010-9452(08)70548-3

Mine, D., \& Yokosawa, K. (2020). Disconnected hand avatar can be integrated into the peripersonal space. Experimental Brain Research. https://doi.org/10.1007/s00221-020-05971-z

Noel, J.-P., Bertoni, T., Terrebonne, E., Pellencin, E., Herbelin, B., Cascio, C., ... Serino, A. (2020). Rapid Recalibration 
of Peri-Personal Space: Psychophysical, Electrophysiological, and Neural Network Modeling Evidence. Cerebral Cortex, 1-19. https://doi.org/10.1093/cercor/bhaa103

Pavani, F., Spence, C., \& Driver, J. (2000). Visual Capture of Touch: Out-of-the-Body Experiences with Rubber Gloves. Psychological Science, 11(5), 353-359. https://doi.org/10.1111/1467-9280.00270

Perez-Marcos, D., Sanchez-Vives, M. V., \& Slater, M. (2012). Is my hand connected to my body? The impact of body continuity and arm alignment on the virtual hand illusion. Cognitive Neurodynamics, 6(4), 295-305. https://doi.org/10.1007/s11571-011-9178-5

Rizzolatti, G., Fadiga, L., Fogassi, L., \& Gallese, V. (1997). The space around us. Science, 277(5323), $190-191$. https://doi.org/10.1126/science.277.5323.190

Serino, A. (2019). Peripersonal space (PPS) as a multisensory interface between the individual and the environment, defining the space of the self. Neuroscience and Biobehavioral Reviews, 99(December 2018), 138-159. https://doi.org/10.1016/j.neubiorev.2019.01.016

Serino, A., Bassolino, M., Farnè, A., \& Làdavas, E. (2007). Extended multisensory space in blind cane users. Psychological Science, 18(7), 642-648. https://doi.org/10.1111/j.1467-9280.2007.01952.x

Serino, A., Canzoneri, E., \& Avenanti, A. (2011). Fronto-parietal areas necessary for a multisensory representation of peripersonal space in humans: An rTMS study. Journal of Cognitive Neuroscience, 23(10), 2956-2967. https://doi.org/10.1162/jocn_a_00006

Serino, A., Noel, J. P., Mange, R., Canzoneri, E., Pellencin, E., Ruiz, J. B., ... Herbelin, B. (2017). Peripersonal space: An index of multisensory body-environment interactions in real, virtual, and mixed realities. Frontiers in ICT, 4(JAN), 1-12. https://doi.org/10.3389/fict.2017.00031

Varnava, A., McCarthy, M., \& Beaumont, J. G. (2002). Line bisection in normal adults: Direction of attentional bias for near and far space. Neuropsychologia, 40(8), 1372-1378. https://doi.org/10.1016/S0028-3932(01)00204-4 